\title{
Comparative Study of Concordance of Digital Models, Plaster Models, and 3D Printed Filament Models with Clinical Measurements in Children
}

\author{
Hugo Ricardo Rosin' Mariana de Oliveira Daltoé1 Carolina Maschietto Pucinelli' Fábio Lourenço Romano \\ Fabrício Kitazono de Carvalho' Léa Assed Bezerra da Silva' Raquel Assed Bezerra Segato'
}

'Department of Pediatric Clinics, School of Dentistry of Ribeirão Preto, University of São Paulo (USP), Ribeirão Preto, São Paulo, Brazil
Address for correspondence: Mariana de Oliveira Daltoé, Avenida do Café, s/n. 14040-904. Ribeirão Preto. SP. Brazil

E-mail: mariana.daltoe@usp.br

\begin{abstract}
Objective: The present study aimed to evaluate, in pediatric patients, the concordance of intraoral scanner for dental measurements, comparing the measurements obtained clinically with digital models, 3D printed filament models, and conventional plaster models.

Materials and Methods: For this study, 31 patients with mixed dentition were selected, with at least the upper central incisors and upper first permanent molars erupted. The dental size measurement obtained with 3Shape Trios Scanner was compared with that obtained clinically with the aid of a digital caliper, as well as the measurements made with plaster models and filament printed models. For data analysis, the intraclass correlation coefficient (ICC) was performed and the agreement was categorized according to it. The Bland-Altman analysis was also applied to the data to graphically display the concordance.

Results: There was no difference in agreement between measurements made in plaster and filament models compared to the reference method, and for measurements in the digital model, the agreement was low or zero in the molar region.

Conclusion: According to the present study, we can conclude that both plaster and filament models presented values that are faithful to those obtained clinically and that the evaluated region affected the agreement with the reference method.

Keywords: Digital molding, intraoral scanning, molding, pediatric dentistry, study models
\end{abstract}

\section{Introduction}

In pediatric dentistry and orthodontics, the use of dental arch plaster models is of fundamental importance in clinical practice, especially for correct diagnosis and planning of malocclusions, ensuring a permanent record of teeth positioning in three dimensions. $[1,2]$ The models allow to verify symmetry and shape of the arches, inclination, anatomy, size, and position of the teeth, as well as to perform different orthodontic analyses related to the arches perimeter.[3] Besides, plaster models are also a valuable form of legal registration [4] and are very useful in comparing the results obtained after performing dental interventions, as well as for the correct transfer of patients between professionals.[5]

How to cite this article: Rosin HR, Daltoé MD, Pucinelli CM, Romano FL, de Carvalho FK, da Silva LAB, Segato RAB. Comparative Study of Concordance of Digital Models, Plaster Models, and 3D Printed Filament Models with Clinical Measurements in Children. J Pediatr Dent 2020;6(1):1-11

OPEN ACCESS This work is licensed under a Creative Commons Attribution-NonCommercial 4.0 International License. 
However, the use of study plaster models has been questioned by the possibility of distortions and lack of accuracy inherent to dental materials.[2] In addition, there are often limitations on the physical storage space, as well as the possibility of cross-contamination. It is noteworthy that the disinfection protocols, besides representing an increase of clinical time, can change the model's dimensional reliability. Further, many professionals neglect this step which puts everyone involved in handling the models at risk. [1]

The discomfort of the molding procedure, in addition to the difficulties in correctly performing work techniques, has been a constant challenge for professionals. [6] Moreover, these techniques, when applied to children or young patients, can cause unwanted reactions, such as nausea and vomiting, which impair the outcomes of the procedure, working time, and professional-patient relationship.[3] In this sense, intraoral scanning represented a great evolution for the area. It presents advantages such as decreased patient discomfort, agility in the clinical professional work, improved communication between colleagues and with the prosthesis and/or orthodontic laboratories, as well as reduction of the physical spaces required for archiving models.[7]

The use of intraoral scanning may also be particularly advantageous for anxious patients prone to nausea episodes during impression as well as for cleft palates who may be at increased risk for aspiration of impression material. [8,9] Further, for patients undergoing orthodontic treatment with fixed appliances, intraoral scanning is a good option as orthodontic devices can damage the impression material when removing the trays, potentially causing changes in the models obtained.[7]

No studies are evaluating the concordance of the images obtained from intraoral scanning and what impact this device would have on child care, specifically in patients in the mixed dentition phase. Thus, the present study aimed to evaluate, in pediatric patients, the concordance of the measurements obtained in a plaster model, measurements in a filament model, and measurements in a digital model obtained by direct intraoral scanning, compared to the measurements obtained clinically.

\section{Materials and Methods}

Initially, the project was submitted to the institutional review board approval (process \# 79668617.2.0000.5419). Patients and guardians were informed about the research project and were included in this study after reading the Research Participant Information Letter and signing the informed consent form.

A total of 31 patients $(n=31)$ who aged $7-12$ years and who met the following inclusion criteria were selected:

1. Mixed dentition with at least fully erupted central incisors and maxillary permanent molars

2. Mouth opening $>25 \mathrm{~mm}$

3. Absence of shape and/or size dental anomalies

4. Absence of extensive caries lesions on the teeth to be analyzed

5. Indication of orthodontic evaluation.

In each patient, the following procedures were performed.

\section{Clinical measurement (reference method)}

With the aid of a digital caliper, measurements were made in the buccolingual direction in the first permanent maxillary molars (D16 and D26), at the height of the marginal crest, and in the mesiodistal direction in the permanent maxillary central incisors at the incisal edge (D11 and D21). These measurements on patients' teeth were considered as the reference method or the "gold standard" ( $n=4 /$ patient).

\section{Intraoral scanning}

Upper arch intraoral scanning was performed with 3Shape Trios Color (3Shape A/S, Copenhagen, Denmark). The tip of the equipment was used to scan the upper arch, respecting the one-way started in the posterior right area, passing through the anterior area, and ending with the posterior left area. This method generated a 3D digital file (in .stl format file).

\section{Obtaining the filament model}

The .stl file obtained through the digital impression was sent to a 3D printer (GTMax3D, Americana/SP, Brazil) to make a filament printed model that physically represented the digital model.

\section{Alginate molding}

For molding and obtaining the conventional model, the following steps were followed:

a. Dental prophylaxis with rubber bowl and pumice stone (Maquira Industry of Dental Products S.A., Maringá, PR, Brazil) and water

b. Selection of tray (Maquira Industry of Dental Products S.A., Maringá, PR, Brazil)

c. Adaptation of utility wax (Wilson-Polidental, Cotia, SP, Brazil) on the edges of the tray for peripheral sealing 
d. Proportion and handling of impression material (Alginate) according to the manufacturer's recommendations (Avagel Dentsply, Catanduva, SP, Brazil)

e. Material insertion into the previously selected tray

f. Insertion of the tray into the patient's oral cavity, respecting the anterior to posterior movement that favors the flow of the impression material

g. Upon completion of the setting of the impression material, the impression was removed, disinfected by spraying with $5 \%$ sodium hypochlorite (Rioquímica, São Jose do Rio Preto, SP, Brazil), and allowed to stand for $10 \mathrm{~min}$. Then, excess sodium hypochlorite was removed with running water

h. Proportion and mixing of type III plaster (Asfer, São Caetano do Sul, SP, Brazil) according to the manufacturer's instructions and model leakage, under mechanical vibration, and in incremental portions, starting with the dental cusps and later for the rest of the teeth and alveolar base.

Clinically performed measurements (reference method) were repeated in digital models using OrthoAnalyzer software (3Shape A/S, Copenhagen, Denmark). A digital impression was used to generate a filament model on a $3 \mathrm{D}$ printer. Thus, the measurements that were performed clinically and also in the digital archives were repeated in the plaster models obtained by alginate molding and in the filament printed models, with the aid of a digital caliper, respecting the same anatomical references.

Measurement data were statistically treated to identify discrepancies between clinical measurements and digital models, filament models, or plaster models.

\section{Statistical analysis}

The analyses were performed using the $\mathrm{R}$ version 3.6.0 program (University of Auckland, New Zealand). The intraclass concordance coefficient (ICC) was used to verify which of the measurements (plaster cast measurements, filament cast measurements, and digital scan measurements) are more consistent with the clinically obtained measurements (reference method), for each evaluated tooth (D11, D21, D16, and D26). The ICC ranges from 0 to 1 , with measurements close to 1 indicating greater agreement. Agreement was categorized according to the ICC value as: null (0), mild or low (0.01-0.20), fair (0.21-0.40), moderate (0.41-0.60), substantial (0.61-0.79), and high (0.80-1.00).

\section{Results}

Data obtained from 31 patients $(n=31)$ were evaluated, being 20 female children and 11 male children (mean age $9.19 \pm 1.30$ years). The ICC was estimated to verify which of the measurements (plaster cast measurements, filament cast measurements, and digital scan measurements) are more consistent with the clinically obtained measurements (reference method), for each evaluated tooth (D11, D21, D16, and D26). The intraclass agreement coefficient (ICC) ranges from 0 to 1 , with measurements close to 1 indicating greater agreement. The results obtained are presented in Table 1.

Scatter and Bland-Altman charts were constructed. The Bland-Altman graph describes the agreement between two observers, evaluators, or methods, considering quantitative variables. The Bland-Altman plot is a scatter plot between the individual averages of the two

Table 1. Results of the intraclass concordance coefficient of the reference method (clinical evaluation) measurements compared the plaster models, filament models, and digital models, in each measured tooth, showing the lower limit and upper limit

\begin{tabular}{|l|c|l|l|l|}
\hline Tooth & Method & ICC & LL & UL \\
\hline 11 & Plaster model & 0.96 & 0.92 & 0.98 \\
\hline & Filament model & 0.97 & 0.93 & 0.98 \\
\hline 21 & Digital model (scanning) & 0.81 & 0.63 & 0.90 \\
\hline & Plaster model & 0.98 & 0.95 & 0.99 \\
\hline & Filament model & 0.97 & 0.94 & 0.99 \\
\hline 16 & Digital model (scanning) & 0.86 & 0.73 & 0.93 \\
\hline & Plaster model & 0.85 & 0.71 & 0.92 \\
\hline 26 & Filament model & 0.95 & 0.89 & 0.97 \\
\hline & Digital model (scanning) & 0.04 & 0.62 & 0.38 \\
\hline & Plaster model & 0.80 & 0.72 & 0.90 \\
\hline
\end{tabular}

Abbreviations: ICC: Intraclass concordance coefficient, LL: Lower limit, UL: Upper limit 
measurements and the individual differences between the measurements.

The measurements from filament models obtained after intraoral scanning were the ones that most agreed with the reference method measurements (Figs. 1-4) for all teeth evaluated with ICC values equal to 0.97 , $0.97,0.95$, and 0.86 for teeth $11,21,16$, and 26 , respectively. Comparison of the measurements obtained from the plaster models (Figs. 5-8) with the reference method indicated lower agreement coefficients for the posterior teeth, with the ICC values being $0.96,0.98$, 0.85 , and 0.80 for teeth $11,21,16$, and 26 , respectively A comparison between the measurements obtained from the digital model with the reference method (Figs. 9-12) indicated lower agreement coefficients with ICC values of $0.81,0.86,0.04$, and zero for teeth $11,21,16$, and 26 , respectively. It can also be verified that, for teeth 16 and 26, there was no agreement of the digital model measurements with the reference method (Fig 11 and 12).
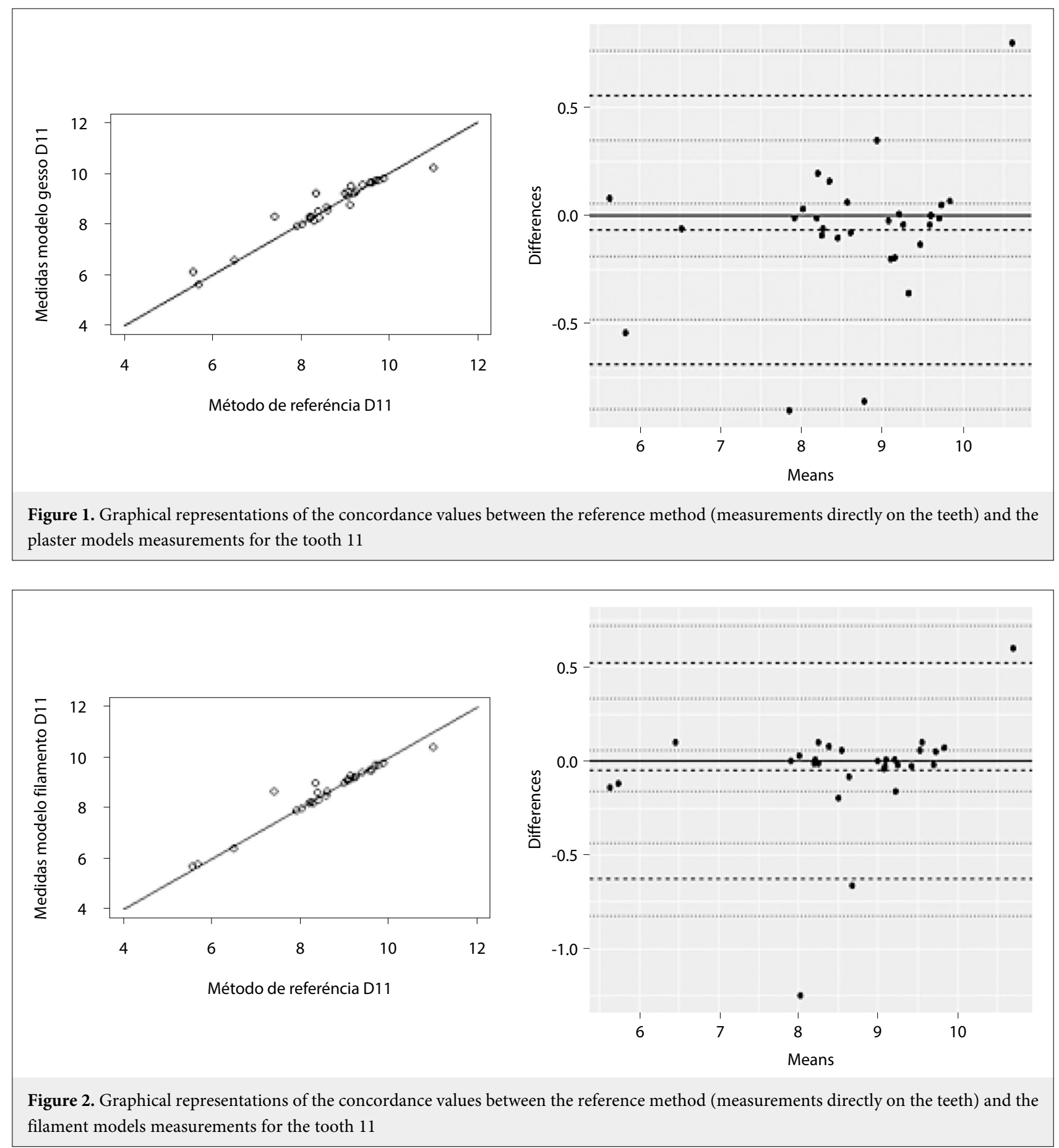

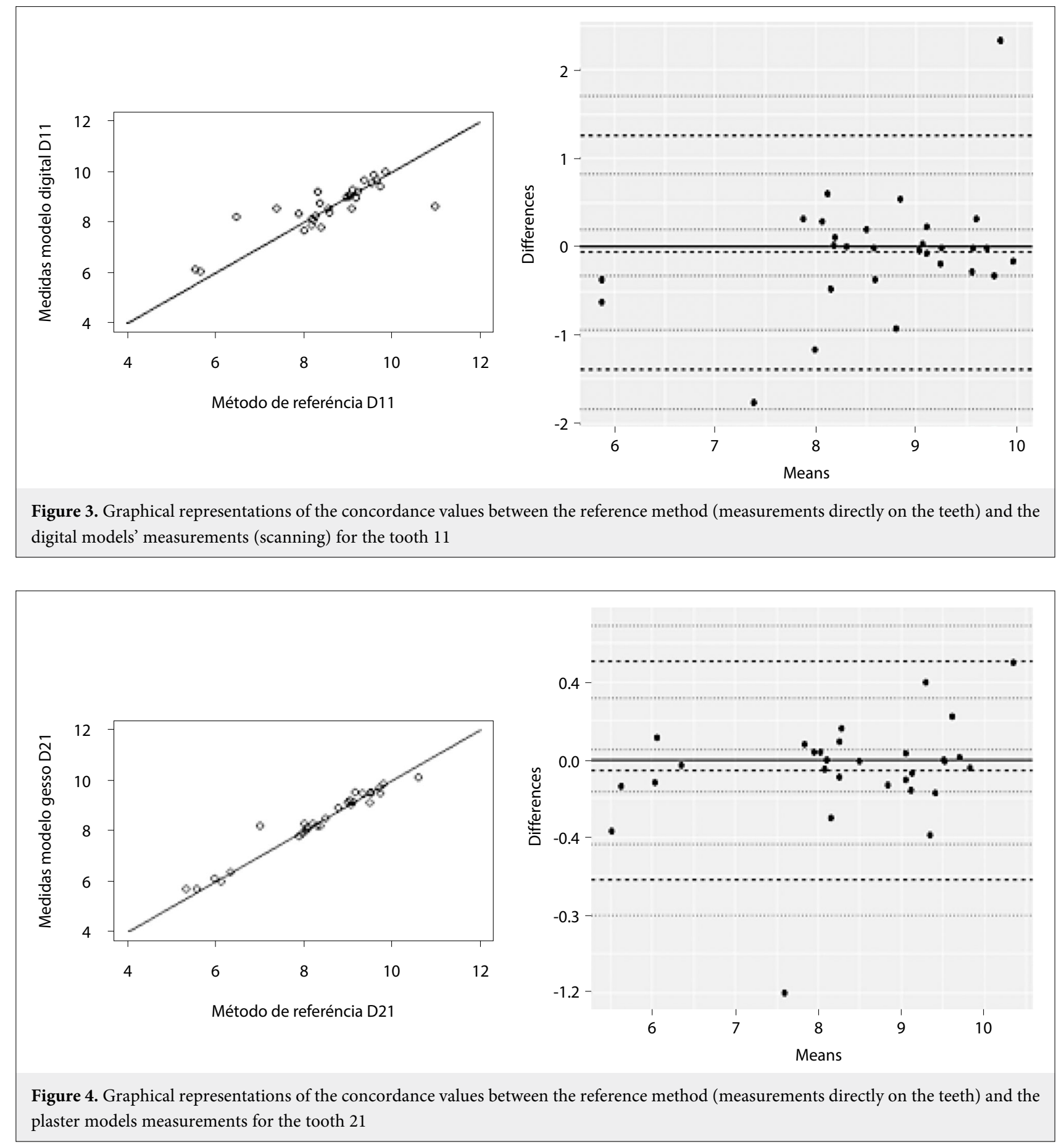

For the comparison between the reference method and the measurements obtained in the filament and plaster models, the resulting concordance was high. Concerning the measurements obtained in the digital models compared to the reference method, the agreement varied from null to high, depending on the evaluated region, being high for the upper central incisors, low for the right first permanent molar, and null for the left first permanent molar, indicating that the evaluated mouth region affected agreement.

\section{Discussion}

Direct intraoral scanning has several advantages when it comes to making digital or even printed models without going through the molding step. In addition to material and time savings, patient comfort is proven to be greater, suggesting this technique as a strong candidate for use in children and young patients. [6] However, to date, there is no work in the literature evaluating the use of intraoral scanning in children. The present study 

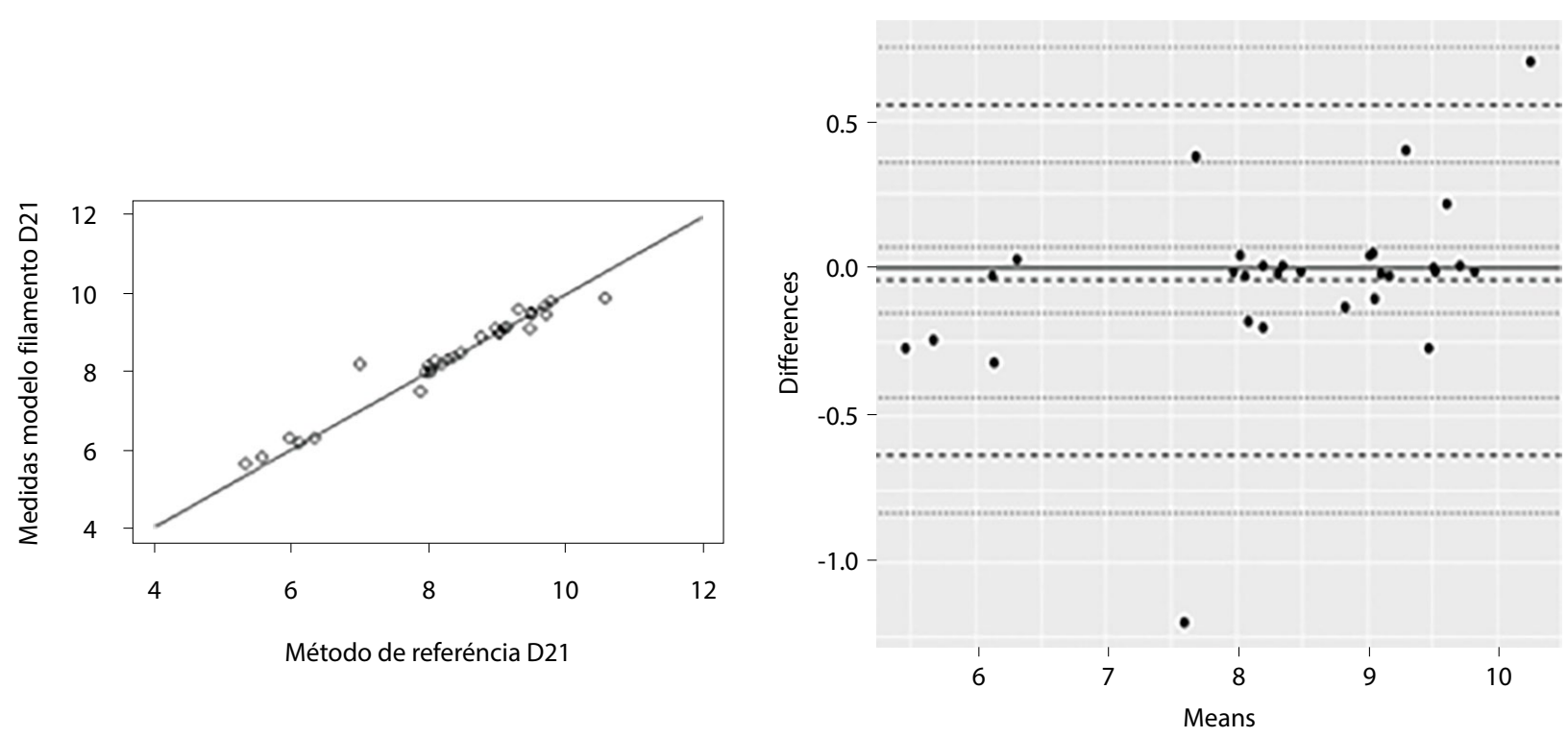

Figure 5. Graphical representations of concordance values between reference method (measurements directly on the teeth) and filament models measurements for the tooth 21

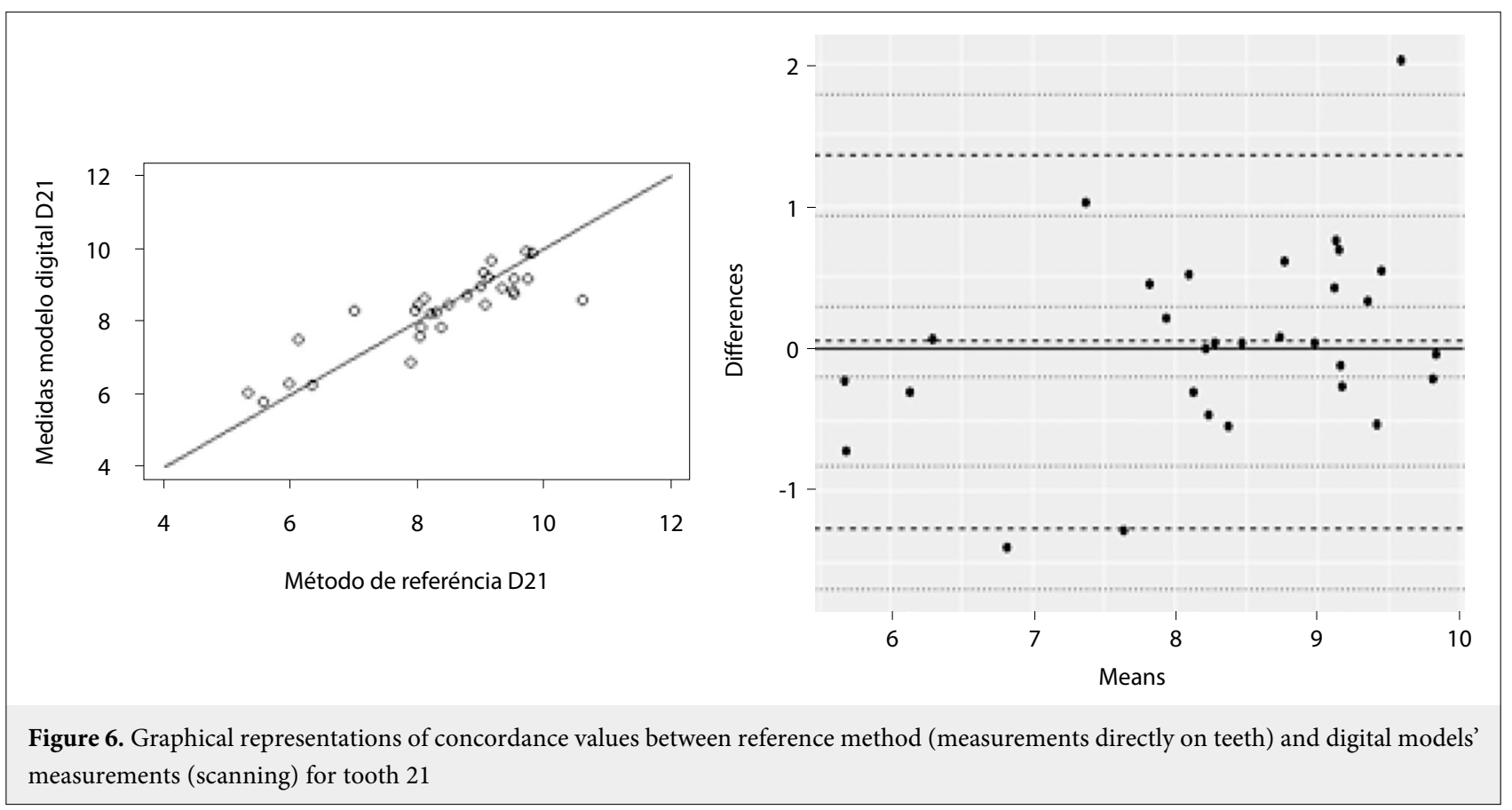

is the first to evaluate the concordance of measurements in different models in mixed dentition patients.

Regarding comfort and preference over different methods, a recent study has shown that digital molding was considered more comfortable compared to the conventional alginate method and was considered the preferred method.[10] This study concluded that there was no statistically significant difference between the conventional molding and digital molding, concerning the time required for the technique.
Tomita et al compared resin models obtained through digital models after direct scanning and digital models obtained after scanning the conventional molding (by alginate and silicone) and the plaster model. The authors found no significant difference after analysis of the different methods for obtaining models.

In 2017, Camardella et al performed a study comparing the measurements on digital models obtained by scanning and the measurements performed on conventional plaster models of 28 volunteers, similar to what 

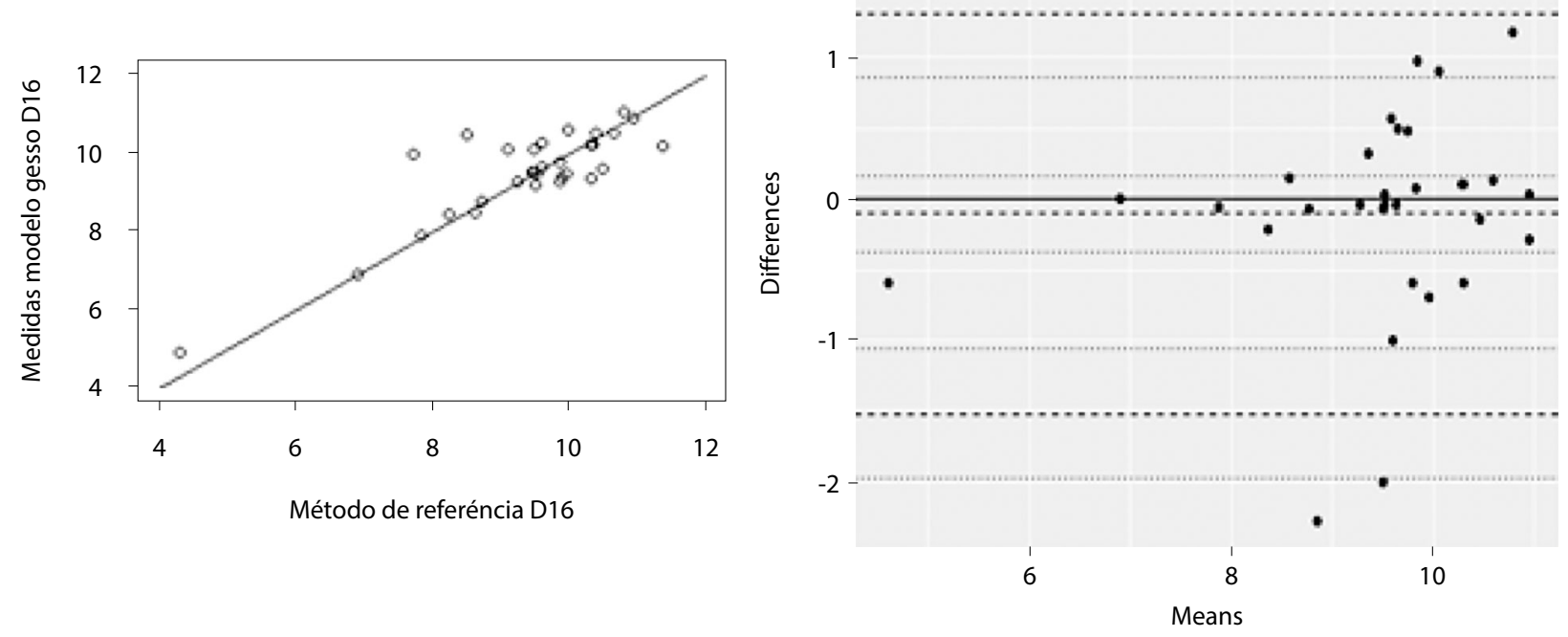

Figure 7. Graphical representations of the concordance values between the reference method (measurements directly on the teeth) and plaster models measurements for tooth 16

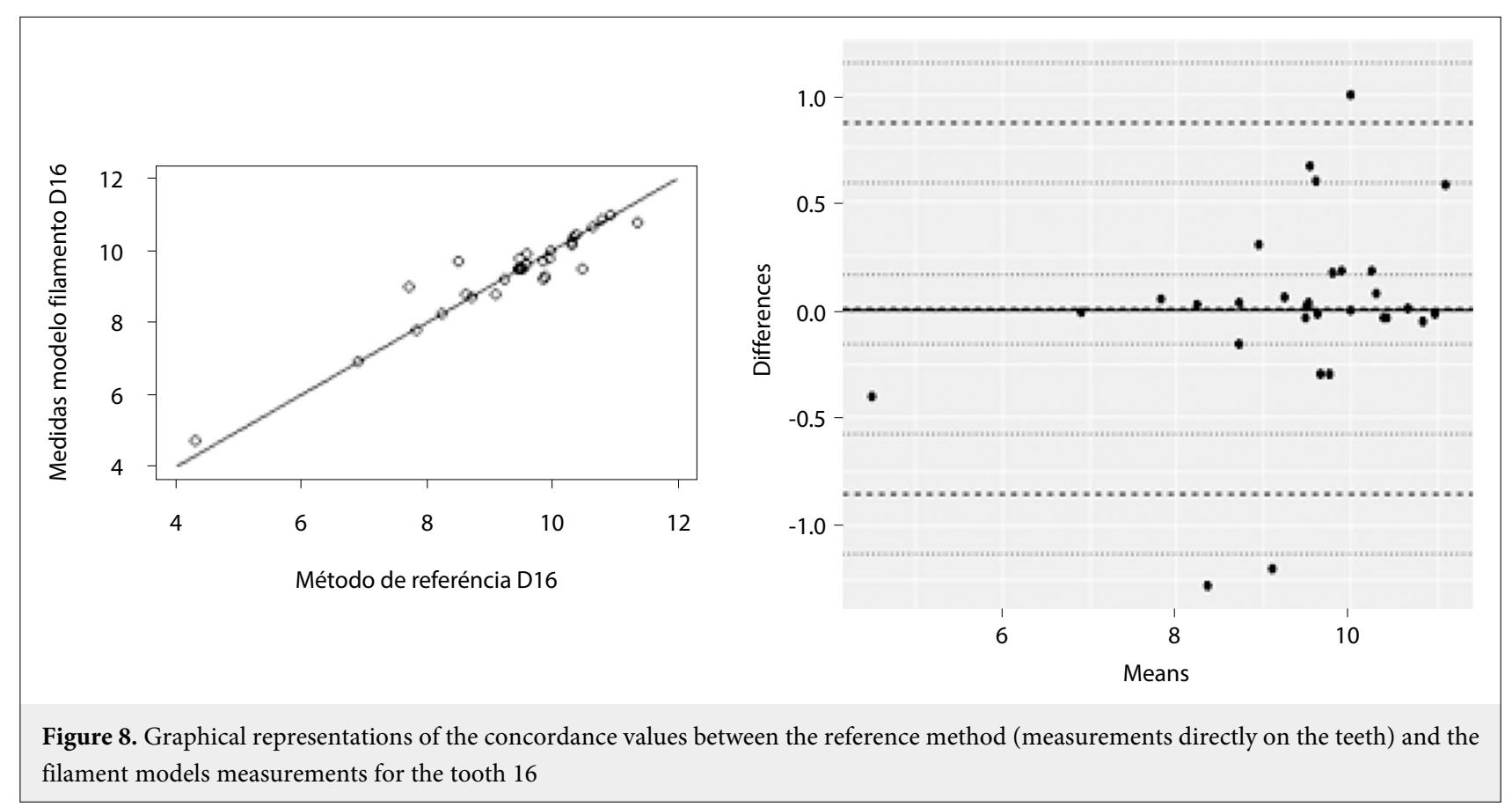

was also done in the present work, but the volunteers were adults. The authors concluded that the measurements obtained with the scanner were clinically acceptable when compared to those obtained in the plaster models, although they were affected by the evaluated region, such as the height of the maxillary central incisors, which showed the highest degree of error. These results differ from those found in the present study since we observed that the lowest concordance was found in the molar region.
Another study evaluating the validity of intraoral scanning in adults compared to plaster models concluded that there was no statistically significant difference between the two methods, except for one measurement, the lower intermolar distance.[11] These results not only coincide with other studies in the literature showing that the greatest distortions occur in the posterior areas of the models[12] but also coincide with the results found in the present study, although we evaluated only the upper arch. Intraoral conditions such as 


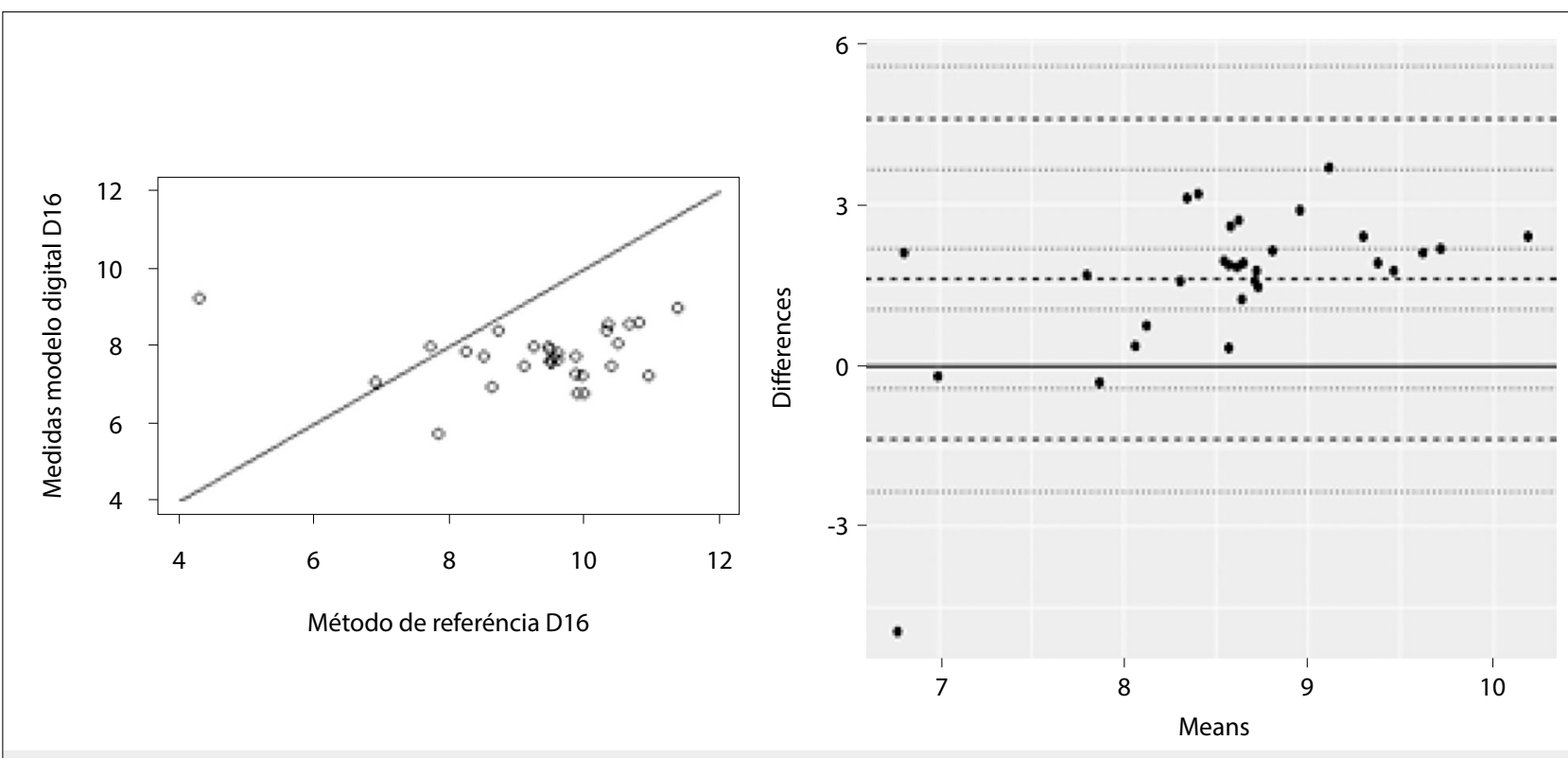

Figure 9. Graphical representations of the concordance values between the reference method (measurements directly on the teeth) and the digital models (scanning) measurements for the tooth 16

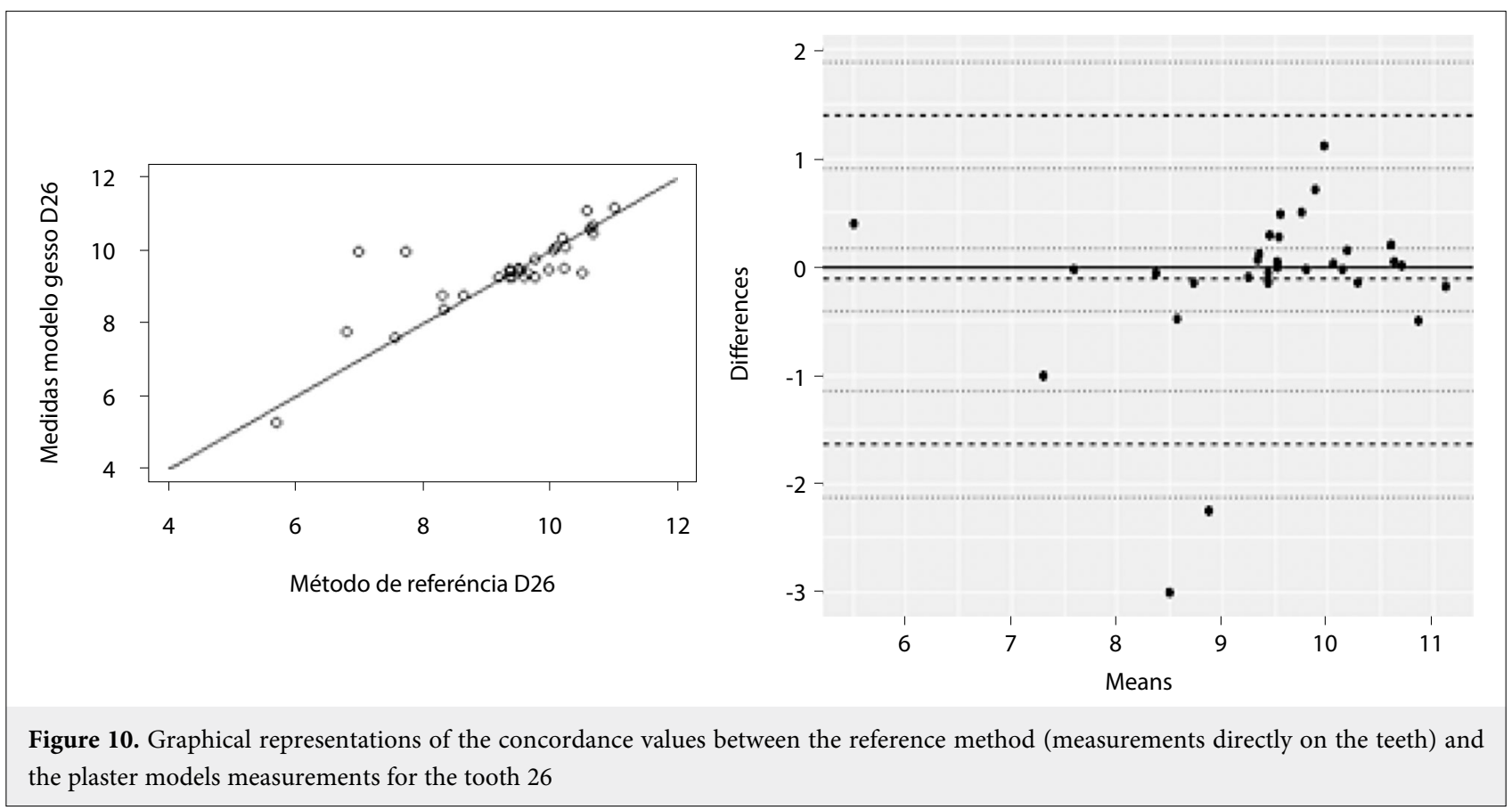

saliva, tongue, and limited arch space are the factors that may contribute to the distortions found in the scans and could lead to greater distortion in the mandibular arch.[11]

It has been shown in the literature that different scanning techniques, changing the direction of the device during the full arch molding procedure, have different effects on the obtained model accuracy, but the different device brands did not affect the final result. [13] Furthermore, another study compared scans from different devices for reproducibility and image veracity, considering the repetition experience. The results showed that the Trios scanner was significantly more accurate about the operators' ability than the other equipment evaluated (iTero). However, image veracity improved over repetition of use with the iTero group, showing that clinical use experience affected the veracity of scanned images, which did not occur with the Trios group. This study also demonstrated that the scanned region affects the accuracy of the obtained 

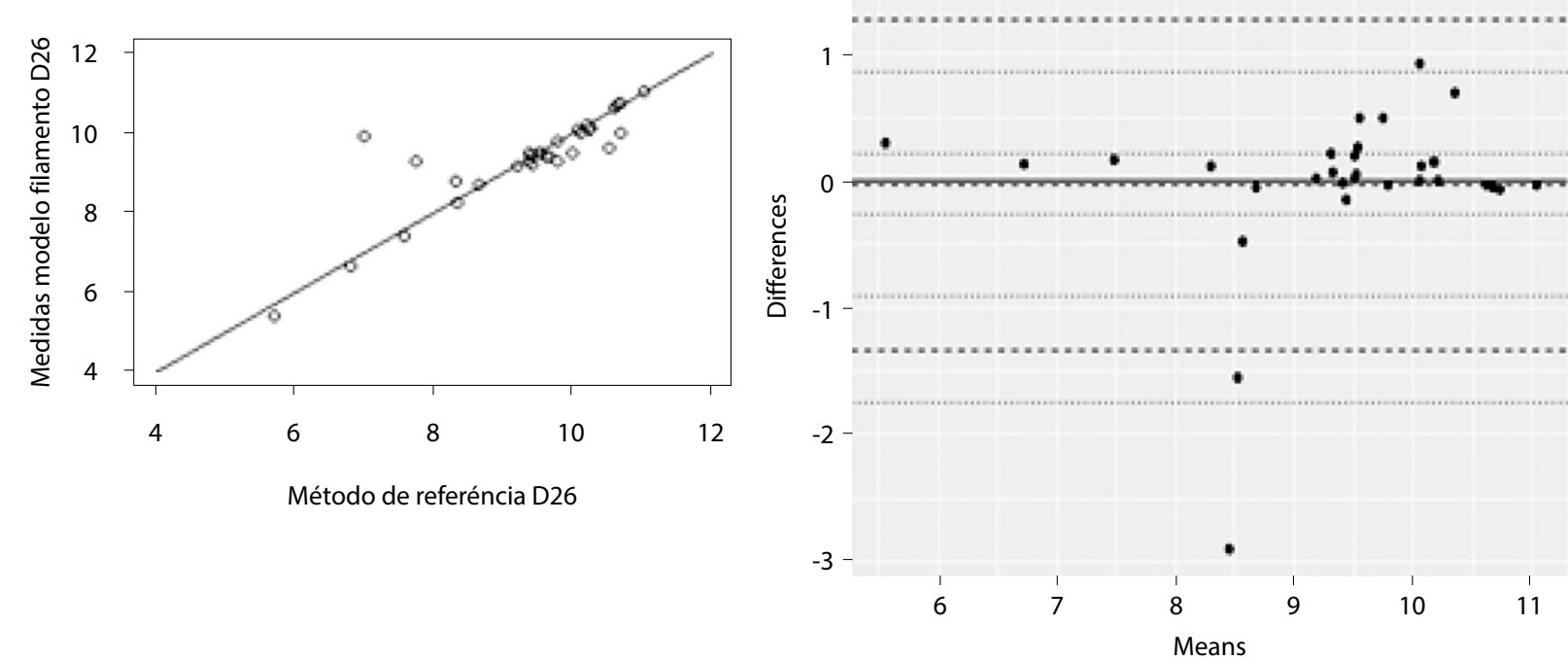

Figure 11. Graphical representations of the concordance values between the reference method (measurements directly on the teeth) and the filament models measurements for the tooth 26
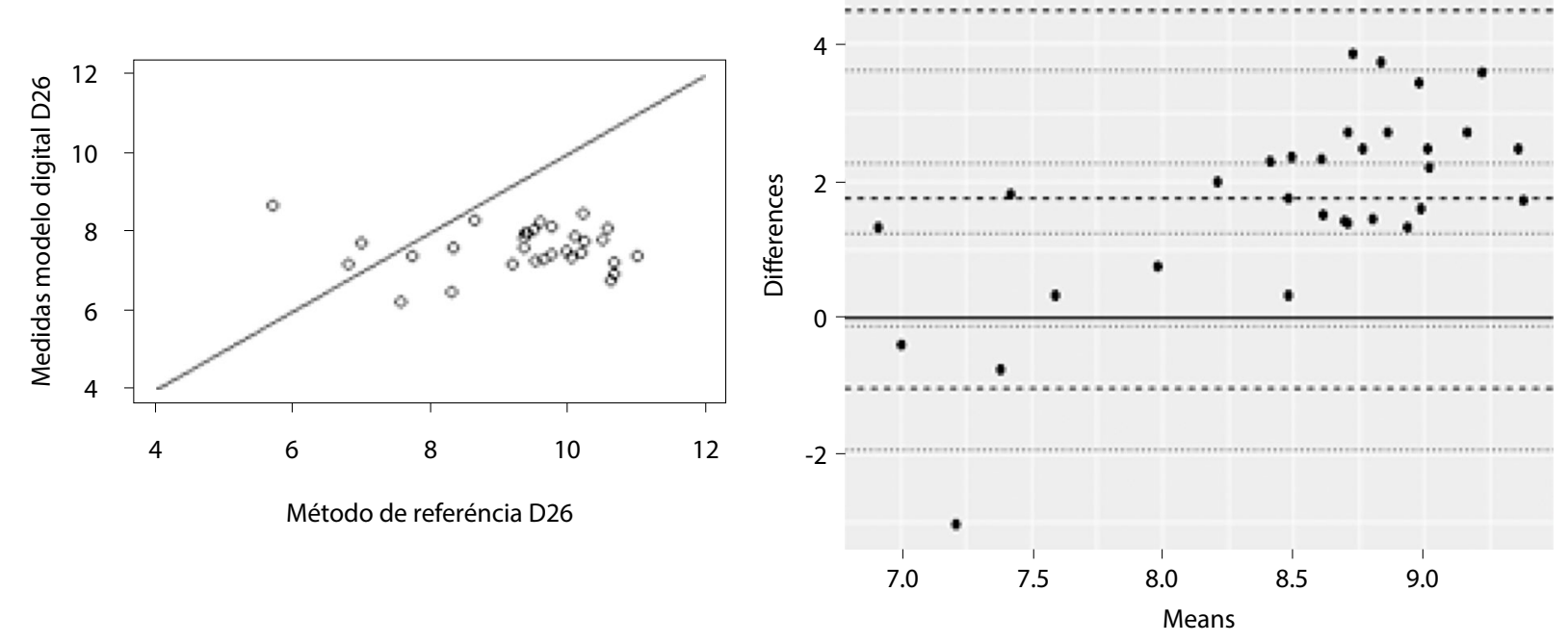

Figure 12. Graphical representations of the concordance values between the reference method (measurements directly on the teeth) and the digital models' measurements (scanning) for the tooth 26

scan, and the maxillary arch showed less distortion than the mandibular arch.[14] In contrast, our study was conducted in the maxillary arch and by an experienced evaluator, which would minimize these interferences in the obtained results.

At the same time, when assessing dental measurements of Bolton diameter and proportions in digital models and plaster models, the largest discrepancies in the upper first molar measurements were observed, similar to the results found in the present study.[15] We agree with these authors who attributed these results to the fact that it is a commonly rotated tooth in the arch, while the central incisors are easier to see.

In the present study, although we found zero concordance for the measurements in the molar region performed on digital models, the same did not occur for the filament models that were obtained through this digital molding. Unlike digital models, the filament models showed high agreement with the reference method measurements, which leads us to believe that 
the digital molding generates a concordant image capable of printing a highly accurate model. However, the digital model does not allow for the same ease of manipulation or requires more experience for manipulation and measurement than a printed physical model. Further studies are needed to verify the influence of operator skill and experience in measuring using digital model software.

Small differences between the different methods are common to be found as several factors contribute to this: such as there is no physical barrier for reference and measurement in virtual models; difficulty in contact point scanning and algorithm filling of these regions; contraction or expansion that may occur in the molding materials; and the clinical experience of the person performing the procedure or measurements. However, these differences are not clinically significant and do not preclude the use of different methods as the studies reported here demonstrate.[6,14-17]

Thus, direct intraoral scanning is an important tool for obtaining study models and analysis of dental measurements for children and young patients, since satisfaction with this method has already been shown to be higher in adults[18] and children.[10] However, more studies are still needed, especially clinical ones, since most studies in the literature analyze the accuracy of this method in resin models[13,17] so that it can be used in the same way as models obtained via conventional molding without harming future clinical conducts.

\section{Conclusion}

Respecting the limitations, we can conclude based on this work results:

1. There was a high concordance between the measurements made in the plaster models obtained via alginate impression and the measurements made in the filament models obtained via digital impression printing, compared to the reference method (intraoral evaluation)

2. For all evaluated methods, concordance with the reference method was high for measurements in the incisor region

3. For measurements in the digital model, concordance with the reference method was lower (low or zero) than that obtained with the other methods, especially in the molar region

4. For the molar region, there was a tendency to decrease concordance in the measurements of the filament and plaster models, denoting greater difficulty for measurements in the posterior region.
Financial Disclosure: Nil.

Conflict of Interest: None declared.

\section{References}

1. Westergard EJ, Romito LM, Kowolik MJ, Palenik CJ. Controlling bacterial contamination of dental impression guns. J Am Dent Assoc 2011;142(11):1269-1274 doi:10.14219/jada. archive.2011.0112

2. Vecsei B, Joós-Kovács G, Borbély J, Hermann P. Comparison of the accuracy of direct and indirect three-dimensional digitizing processes for CAD/CAM systems - An in vitro study. J Prosthodont Res 2017;61(2):177-184 doi:10.1016/j.jpor.2016.07.001

3. Goracci C, Franchi L, Vichi A, Ferrari M. Accuracy, reliability, and efficiency of intraoral scanners for full-arch impressions: a systematic review of the clinical evidence. Eur J Orthod 2016;38(4):422-428 doi:10.1093/ejo/cjv077

4. Jiang T, Lee SM, Hou Y, Chang X, Hwang HS. Evaluation of digital dental models obtained from dental cone-beam computed tomography scan of alginate impressions. Korean J Orthod 2016;46(3):129-136 doi:10.4041/kjod.2016.46.3.129

5. White AJ, Fallis DW, Vandewalle KS. Analysis of intra-arch and interarch measurements from digital models with 2 impression materials and a modeling process based on cone-beam computed tomography. Am J Orthod Dentofacial Orthop 2010;137(4):456. e1-457 doi:10.1016/j.ajodo.2009.09.019

6. Burhardt L, Livas C, Kerdijk W, van der Meer WJ, Ren Y. Treatment comfort, time perception, and preference for conventional and digital impression techniques: A comparative study in young patients. Am J Orthod Dentofacial Orthop 2016;150(2):261-267 doi:10.1016/j.ajodo.2015.12.027

7. Akyalcin S, Cozad BE, English JD, Colville CD, Laman S. Diagnostic accuracy of impression-free digital models. Am J Orthod Dentofacial Orthop 2013;144(6):916-922 doi:10.1016/j. ajodo.2013.04.024

8. Chalmers EV, McIntyre GT, Wang W, Gillgrass T, Martin $\mathrm{CB}$, Mossey PA. Intraoral 3D scanning or dental impressions for the assessment of dental arch relationships in cleft care: Which is superior?. Cleft Palate Craniofac J 2016;53(5):568-577 doi: $10.1597 / 15-036$

9. Choi YS, Shin HS. Preoperative planning and simulation in patients with cleft palate using intraoral three-dimensional scanning and printing. J Craniofac Surg 2019;30(7):2245-2248 doi:10.1097/SCS.0000000000005983

10. Yilmaz H, Aydin MN. Digital versus conventional impression method in children: Comfort, preference and time. Int J Paediatr Dent 2019;29(6):728-735 doi:10.1111/ipd.12566

11. Zhang F, Suh KJ, Lee KM. Validity of intraoral scans compared with plaster models: An in-vivo comparison of dental measurements and 3D surface analysis. PLoS One 2016;11(6):e0157713 doi:10.1371/journal.pone.0157713

12. Patzelt SB, Bishti S, Stampf S, Att W. Accuracy of computer-aided design/computer-aided manufacturing-generated dental casts based on intraoral scanner data. J Am Dent Assoc 2014;145(11):1133-1140 doi:10.14219/jada.2014.87 
13. Favero R, Volpato A, Francesco M, Fiore AD, Guazzo R, Favero L. Accuracy of 3D digital modeling of dental arches. Dental Press J Orthod 2019;24(1):38e1-37e7 doi:10.1590/2177-6709.24.1.38. e1-7.onl

14. Lim JH, Park JM, Kim M, Heo SJ, Myung JY. Comparison of digital intraoral scanner reproducibility and image trueness considering repetitive experience. J Prosthet Dent 2018;119(2):225232 doi:10.1016/j.prosdent.2017.05.002

15. Naidu D, Freer TJ. Validity, reliability, and reproducibility of the iOC intraoral scanner: a comparison of tooth widths and Bolton ratios. Am J Orthod Dentofacial Orthop 2013;144(2):304-310 doi:10.1016/j.ajodo.2013.04.011
16. Camardella LT, Breuning H, de Vasconcellos Vilella O. Accuracy and reproducibility of measurements on plaster models and digital models created using an intraoral scanner. J Orofac Orthop 2017;78(3):211-220 doi:10.1007/s00056-016-0070-0

17. Tomita Y, Uechi J, Konno M, Sasamoto S, Iijima M, Mizoguchi I. Accuracy of digital models generated by conventional impression/plaster-model methods and intraoral scanning. Dent Mater J 2018;37(4):628-633 doi:10.4012/dmj.2017-208

18. Burzynski JA, Firestone AR, Beck FM, Fields HW Jr, Deguchi T. Comparison of digital intraoral scanners and alginate impressions: Time and patient satisfaction. Am J Orthod Dentofacial Orthop 2018;153(4):534-541 doi:10.1016/j.ajodo.2017.08.017 